\title{
VLBI AND EXTREME SCATTERING EVENTS
}

\author{
Brian Dennison \\ Physics Department \\ Virginia Polytechnic Institute and State University \\ Blacksburg, VA 24061 USA \\ R. L. Fiedler and K. J. Johnston \\ E. O. Hulburt Center for Space Research \\ Code 4130 \\ Naval Research Laboratory \\ Washington, DC 20375 USA
}

\begin{abstract}
Ray tracing numerical simulations of extreme scattering events result in light curves that display many of the qualitative features observed in such events. The apparent images of an affected source show strong and variable distortions, which could be readily discerned with VLBI observations.
\end{abstract}

An extreme scattering event (ESE) is evidently caused by an interruption of the line of sight to a compact source by a dense compact structure in the interstellar medium (Fiedler et al. 1987a,b). The resulting refraction produces strong fluctuations in the received flux density. An affected source should therefore exhibit major, and variable, distortions in its apparent structure, during the ccurse of such an event.

To gain a qualitative understanding of the appearance of an affected source, ray tracing calculations were carried out for various types of intervening ionized clouds. The background source was assumed to be a circularly symmetric Gaussian, with a half-power diameter of 0.8 mas. The calculations were carried out in the limit of geometric optics, with the refraction angles calculated from the impulse approximation.

A model that qualitatively reproduces many of the observed features of the ESE seen in 0954 + 658 (Fiedler et al. 1987a,b) involves a highly irregular cloud, in which the two-dimensional column density profile was synthesized from 12 randomly chosen Gaussian distributions. The overall properties of the cloud are given in Table 1.

The apparent brightness distributions, and light curves (Figure 1), were computed for a range of impact parameters and at two frequencies $(2.7$ and $8.1 \mathrm{GHz})$. Not surprisingly, major distortions were present, including multiple imaging and elongations. In Figure 2, we show contour diagrams of the apparent brightness distribution at $8.1 \mathrm{GHz}$, for a range of offsets between the source and cloud center. At $2.7 \mathrm{GHz}$, the apparent brightness distribution consists of the source plus scattered radiation just before and after the event, and scattered radiation during the event.

We conclude that synoptic VLBI observations, conducted during the course of an event, can directly test the refractive focusing model for ESE's and provide important information on the structure of the refracting clouds. 


\section{Table 1}

Irregular Cloud Model

Mean column density $=5 \times 10^{15} \mathrm{~cm}^{-2} \quad$ Distance $=1000 \mathrm{pc}$

RMS column density $=1.4 \times 10^{16} \mathrm{~cm}^{-2} \quad$ Approximate Diameter $=6$ A.U.

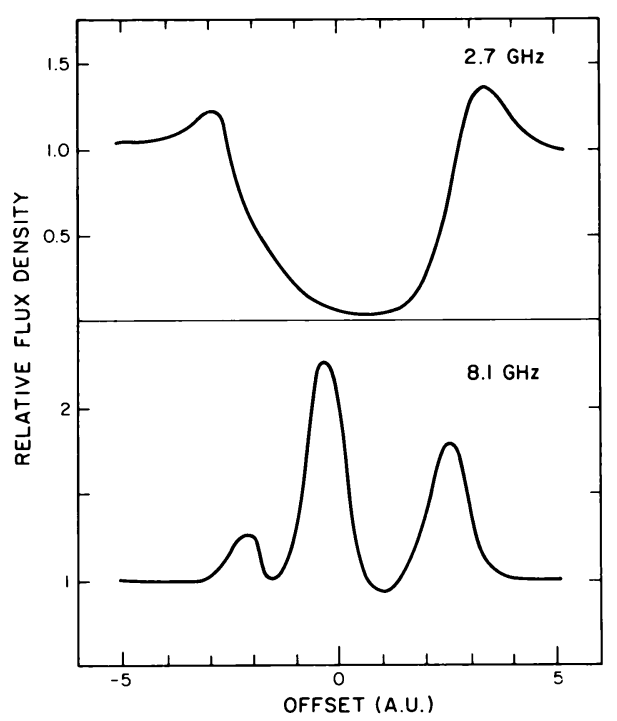

Figure 1. Light curves of a simulated ESE.

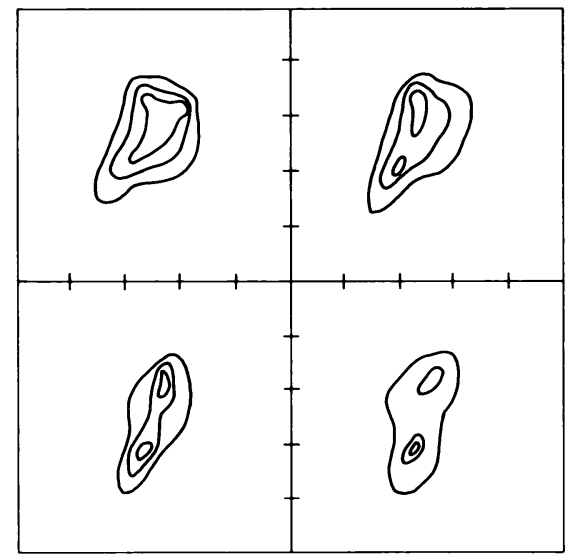

Figure 2. Contours of apparent brightness at $8.1 \mathrm{GHz}$, for source-cloud center offsets of (left to right from upper left) $-0.5,0,0.5,1.0$ A.U. Spacing between tick marks equals 1 mas (equivalent to 1 A.U. at the cloud).

\section{REFERENCES}

Fiedler, R. L., Dennison, B., Johnston, K. J., and Hewish, A. 1987a, Nature, 326, 675.

Fiedler, R. L., Dennison, B., and Johnston, K. J. 1987b, this symposium. 